\title{
Microscopic choked flow for a highly compressible gas in porous media
}

\author{
Hailong Jiang ${ }^{1}$, Yihua Dou ${ }^{1 *}$, Zhongchen $\mathrm{Xi}^{2}$, Mian Chen ${ }^{3}$, Yan Jin ${ }^{3}$ \\ 1 College of Mechanical Engineering, Xi'an Shiyou University, Xi'an 710065, China \\ 2 CCDC Changqing Downhole Technology Company, Xi'an 710018, China \\ 3 State Key Laboratory of Petroleum Resources and Prospecting, China University of Petroleum, Beijing 102249, China \\ *Corresponding author. E-mail address: douyhxapi@163.com
}

\begin{abstract}
Choked flow can impact the gas flow rate from a high-pressure gas well with a vertical fracture of finite conductivity and the development of tensile stress near the wellbore. Traditionally, the choking condition of the flow of a highly compressible gas in porous media is obtained by considering the porous media to be a homogeneous porous medium at the macroscopic scale. In reality, when the average existing pressure of the porous medium decreases, if the compressible gas flow is choked in only one microscopic basic structural unit, the gas flow is choked in the macroscopic porous medium. In this paper, the choking condition of a compressible gas flow in a basic structural unit is studied. It is shown that for the given inlet pressure and temperature, the choked flow occurs first in the basic structural unit with a constant cross-section and with lower porosity and shorter flow distance. If the roughness of the basic structural unit is more complicated or its flow distance is shorter, this basic structural unit requires a lower pressure drop when the gas flow is choked. Whether the basic structural unit is a pipe with finite wall thickness or a single pore, the choking condition first occurs in the position with the smallest porosity or permeability near the exit. It is found that for microscopic choked flow, the outlet-to-inlet pressure under conditions of varying friction is substantially lower than that under the effect of constant friction.
\end{abstract}

Keywords: compressible gas; acceleration; microscopic basic structural unit; choked flow 


\section{Introduction}

The Darcy-Forchheimer equation is given by the appropriate square velocity modification to Darcy's law (Joseph et al., 1982). This modification has been discussed in detail for nearly incompressible flows by Du Plessis and Masliyah (1988), Du Plessis (1994), Hayes et al., (1995), Skjetne and Auriault (1999), Handren et al. (2001), Wang (2012), Wang et al. (2014), and Ai et al. (2015). For compressible flows, the Darcy-Forchheimer equation with an acceleration term is adequate. It is noted that choked flow will occur in the Darcy-Forchheimer equation with an acceleration term (Shreeve, 1968; Emanuel and Jones, 1968; Meyer and Smith, 1985; Nield, 1994; Kodres, 1994; Levy et al., 1995; De Ville, 1996; Ciarletta and Straughan, 2006; Straughan, 2008; Jin et al., 2012a, b; Jiang et al., 2015a, b, c). In these cited works, choked flow is simulated by the averaged Darcy-Forchheimer equation with an acceleration term at the macroscopic scale. In previous studies on choked flows at a macroscopic scale, the choking condition can reduce the gas flow rate in a high-pressure gas well with a finite conductivity vertical fracture (Jiang, et al., 2015c) and increase the risk of tensile failure near the wellbore (Jin, et al., 2011). However, it matters that the macroscopic choked flow and the microscopic choked flow have minute differences when the gas flows through nonhomogeneous porous media, i.e., the microscopic choking condition is different from the macroscopic choking condition. Therefore, it is important to understand what the choking condition is and its influence rule induced by the geometric shape parameters of the basic structural unit at a microscopic scale.

Recently, Yuan (2013) and Yuan et al. (2016) studied the choked gas flow at the pore scale. Macroscopic choked flow can occur when flow in just one pore is choked. It is noted that this research finding was obtained under the effect of constant friction. However, the friction factor cannot be a constant, and it will change with varying gas velocity. The friction factor has a significant effect on the outlet pressure in macroscopic choked flows (Jiang et al., 2015a, b). In this paper, we investigate the relation between the critical condition in which the flow is choked in macroscopic 
porous media and the microscopic basic structural unit under varying friction, where the microscopic basic structural unit is a pipe with finite wall thickness. We prove that when gas flows through a homogeneous porous medium, the critical conditions of choked flow in a macroscopic porous medium and a microscopic basic structural unit are similar, but they are different when the gas flows through a nonhomogeneous porous medium. To elucidate the critical condition at which the flow becomes choked, the geometrically simpler linear adiabatic flow is studied. We explore how the length, the inner radius and the spatial periods of the basic structural unit affect the choking condition. Finally, it is shown that for the same inlet pressure, the outlet pressure under conditions of varying friction is substantially smaller than it is under the constant friction at the choking condition.

\section{Description of porous medium flow}

It is assumed that the void space is an axisymmetric pore throat whose geometrical feature is a circular tube, and the axis of the pore throat is parallel to the $\mathrm{X}$-axis (see Fig. 1). If the geometric shape of all pore throats in a porous medium is the same, the basic structural unit which is a pipe with finite wall thickness can be used to represent the porous medium (see Fig. 2). The inner radius and the outer radius of the basic structural unit are $\mathrm{r}$ and $\mathrm{R}$, respectively. A similar model has been utilized by several authors (Van Golf-Racht, 1982; Coulaud, et al., 1988; Ma and Ruth, 1993; Chukwudozie, et al., 2012). The gas flow is the geometrically simpler linear adiabatic flow. We will employ the ideal gas equation due to its simplicity:

$$
p^{*}=\rho R^{*} T
$$

where $R^{*}$ is the gas constant; and $p^{*}, \rho$ and $T$ are the pressure, density and temperature of the gas, respectively. 

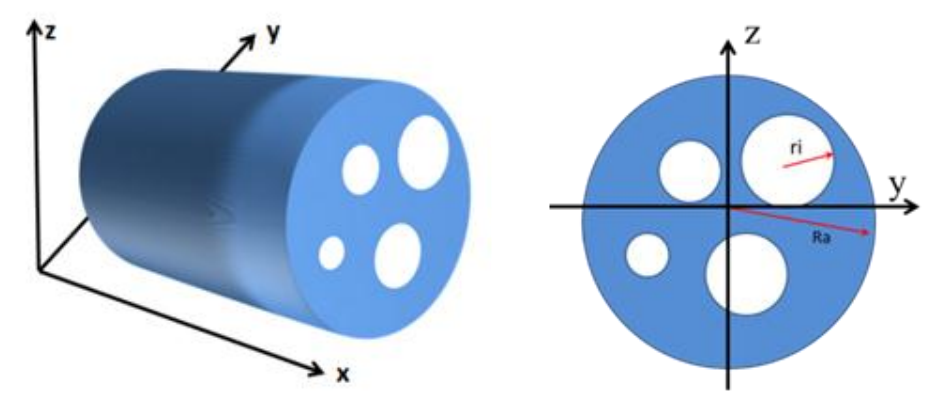

Fig. 1 Schematic diagram of porous media
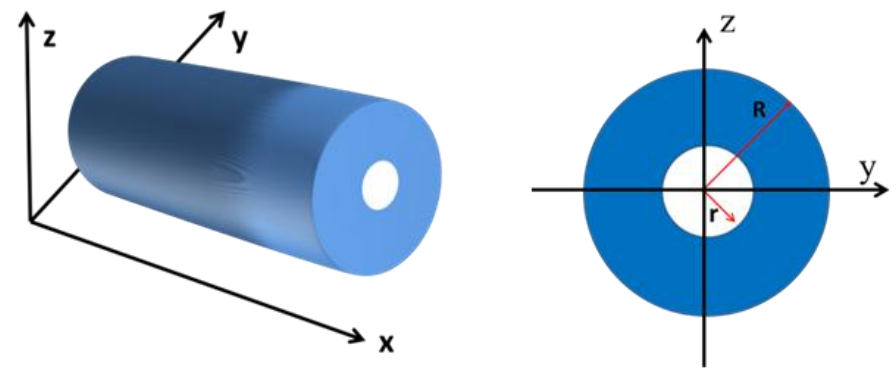

Fig. 2 Schematic diagram of the basic structural unit

The porosity of the basic structural unit $\phi=\left(\frac{r}{R}\right)^{2}$

Van Golf-Racht (1982) derived the permeability of a hydrodynamic unit based on the classic Darcy definition. Similarly we can derive the permeability of the basic structural unit using the same method.

The flow rate of the basic structural unit is like that of a cylindrical capillary,

$$
Q=\frac{\pi r^{4}}{8 \mu} \frac{\Delta p}{\Delta L}
$$

where $\frac{\Delta p}{\Delta L}$ is the pressure gradient in the flow direction, and $\mu$ is the gas viscosity.

The flow rate of the basic structural unit based on the definition of Darcy is

$$
Q=\pi R^{2} \frac{\kappa}{\mu} \frac{\Delta p}{\Delta L}
$$

where $\kappa$ is the permeability of the basic structural unit.

If equation (3) and equation (4) are compared, the following expression will be obtained, 


$$
\kappa=\frac{r^{4}}{8 R^{2}}=\frac{1}{8} r^{2} \phi
$$

\section{The relation of choked flow in a porous medium and the basic structural unit}

The gas flow becomes choked when the average pressure gradient $\left(\frac{d p_{\text {average }}}{d x}\right)$ of the porous medium tends to infinite (Jin, et al., 2012a). There is a difference between the average pressure gradient of the porous medium and the pressure gradient of the basic structural unit. We investigate the reasons for this below.

\subsection{Choked flow in a homogeneous porous medium}

A homogeneous porous medium is one with same inner radius of all its basic structural units in this paper. To simplify the analysis, we assume that the number of basic structural units constituting the homogeneous porous medium is $\mathrm{N}$. The radii of the basic structural unit and the porous medium are $R$ and $R_{a}$, respectively. It is obvious that $R=\frac{R_{a}}{\sqrt{N}}$. The pressures $p$ and $p_{\text {average }}$ are the average pressures of the basic structural unit and homogeneous porous medium, respectively. The average pressure gradient across the homogeneous porous medium is:

$$
\frac{d p_{\text {average }}}{d x}=\frac{d \frac{N \pi R^{2}}{\pi R_{a}{ }^{2}}}{d x}=\frac{d p}{d x}
$$

Because the average pressure gradient of the basic structural unit in the flow direction increases unbound $\left(\frac{d p}{d x} \rightarrow-\infty\right)$, the average pressure gradient of a homogeneous porous medium in the flow direction becomes unbound $\left(\frac{d p_{\text {average }}}{d x} \rightarrow-\infty\right)$. Namely, there is no difference between the macroscopic choked flow in the porous medium and the microscopic choked flow in the basic structural unit.

\subsection{Choked flow in nonhomogeneous porous media}

A nonhomogeneous porous medium in this paper is one with several inner radius 
ranges of the basic structural unit. In the same way, we still assume that the number of basic structural units consisting of nonhomogeneous porous media is N. For those basic structural units, one-half have the characteristic of inner radius as $r_{1}$ and pressure as $p_{1}$ and the other half have an inner radius of $r_{2}\left(r_{1}\right.$ is not equal to $\left.r_{2}\right)$ and pressure as $p_{2}$. Then, the average pressure gradient of a nonhomogeneous porous medium is:

$$
\frac{d p_{\text {average }}}{d x}=\frac{d \frac{N \pi R^{2}\left(p_{1}+p_{2}\right)}{2 \pi R_{a}^{2}}}{d x}=\frac{N}{2}\left(\frac{d p_{1}}{d x}+\frac{d p_{2}}{d x}\right)
$$

Equation (7) shows if that either $\frac{d p_{1}}{d x}$ or $\frac{d p_{2}}{d x}$ increases unbound, then the average pressure gradient of the nonhomogeneous porous medium would surely become unbound. In fact, only one pressure gradient of the basic structural unit can become unbound early when the gas starts to flow. At this time, the macroscopic flow in the nonhomogeneous porous medium shall be considered choked. Traditionally, the critical condition of macroscopic choke flow is obtained by considering the nonhomogeneous porous medium as the homogeneous one. This method clearly is not suitable for the critical condition of macroscopic choked flow in nonhomogeneous porous media. Therefore, the substantial choking condition of macroscopic flow in nonhomogeneous porous media can only be obtained by analyzing the basic structural unit. We investigate the critical condition of choked flow in the basic structural unit below.

\section{Critical condition of choked flow in a basic structural unit}

In this section, we explore the critical condition of choked flow in a basic structural unit at the microscopic scale by adopting the two equations (equation (2) and equation (3)) to estimate the permeability and porosity of the basic structural unit. The flow is adiabatic. The parameters used in these discussions are listed in Table 1.

\subsection{Choked flow in a basic structural unit with constant cross-section in the x-direction}

Assuming that the inner radius of the basic structural unit is constant in the 
$\mathrm{x}$-direction in the basic structural unit, this section provides the impact of the radius and flow distance on critical conditions for choked flow. The gas momentum equation for one-dimensional compressible steady flow is (Nield, 1994)

$$
\frac{\rho}{\phi^{2}} v \frac{d v}{d x}=-\frac{d p}{d x}-\frac{\mu}{\kappa} v-c_{F} \kappa^{-0.5} \rho(v)^{2}
$$

The continuity equation is:

$$
G=\rho v A=\text { constant }
$$

where $\mathrm{A}$ is the effective area of flow, $A=\pi r^{2}$.

Combining Eq. (1), Eq. (8) and Eq. (9), the governing equation is given by the following equation.

$$
\frac{d M a^{2}}{d x}=\frac{\left(2 \phi^{2} M a^{2}+(\gamma-1) M a^{4}\right)}{\left(\phi^{2}-M a^{2}\right)} \gamma M a^{2}\left(\frac{\mu A}{\kappa G}+c_{F} \kappa^{-0.5}\right)
$$

where $M a=\frac{v}{\sqrt{\gamma R^{*} T}}$ is the local Mach number $; \gamma$ is the adiabatic index, which is a constant; $c_{F}$ is a dimensionless coefficient; here $c_{F}=0.55$ (Ward, 1964). The gas pressure gradient can then be determined from

$$
\frac{1}{p} \frac{d p}{d x}=-\frac{\left(\phi^{2}+(\gamma-1) M a^{2}\right)}{\left(\phi^{2}-M a^{2}\right)} \gamma M a^{2}\left(\frac{\mu A}{\kappa G}+c_{F} \kappa^{-0.5}\right)
$$

Equation (10) shows that the local Mach number increases monotonically with the flow of gas in the basic structural unit, and it reaches its maximum value at the exit. Equation (11) shows the pressure gradient at the exit becoming unbounded, which is $\left.\frac{d p}{d x}\right|_{x=L}=-\infty$, when the maximum Mach number at the exit is equal to the porosity. At this critical condition, where the gas flow becomes choked, the boundary conditions of equation (10) and equation (11) are:

$$
\begin{aligned}
& x=0 ; p=p_{\text {inlet }} \\
& x=L ; M a=\phi
\end{aligned}
$$

Firstly, the inlet pressure is given. Secondly, the initial value of the inlet Mach number is given. Thirdly, the inlet Mach number is increased until the ratio between 
the exit Mach number and the exit porosity is near 1. At this point, we can obtain the appropriate Mach number distribution for the microscopic choked flow. Combining the continuity equation, the inlet pressure and this Mach number distribution, we can obtain the gas pressure distribution.

Firstly, the rate of flow at a distance is fixed, $L=1 \mathrm{~m}$, and $r_{0}$ in three models are $0.001 \mathrm{~m}, 0.002 \mathrm{~m}$ and $0.005 \mathrm{~m}$, respectively. When the three models have the same entrance pressure and temperature, Fig. 3 and Fig. 4 show the Mach number distribution and accompanying pressure distribution, respectively, in the flow direction for each of these three basic structural units when the flow is choked. Fig. 3 shows that the choking condition occurs at the exit along the basic structural unit with constant cross-section for each of those three models. Fig. 4 shows that the exit pressure of the basic structural unit with a smaller inner radius is lower when the flow is choked. In other words, the basic structural unit having the smaller inner radius is the first to become choked as the average exist pressure in porous media decreases, and thus the macroscopic flow in the porous medium is also choked. However, this choked condition will not be detected from the macroscopic flow in porous media.

Secondly, Figs. 5 and 6, respectively show the Mach number distribution and accompanying pressure distribution with the same entrance pressure and temperature in the flow direction when the flow is choked. In each figure, the inner radius of the basic structural unit is fixed at $\mathrm{r}_{0}=0.001 \mathrm{~m}$, and $\mathrm{L}$, displayed with three different colors, is given the values of $1 \mathrm{~m} 5 \mathrm{~m}$ and $10 \mathrm{~m}$. It is obvious that the choking condition still occurs in the basic structural unit with a constant cross-section in Fig. 5. Fig. 6 shows the exit pressure of the longer basic structural unit is lower when the flow is choked. This is mainly because a longer basic structural unit allows the pressure loss to be greater because of the drag.

From the discussion above, it can be seen that the choking condition occurs at the exit of the basic structural unit. The choked flow occurs first at the basic structural unit with a constant cross-section, lower porosity and shorter flow distance. 
Table 1. Parameters used in Figs. 3-6 and Figs. 8-15. Adiabatic index $\gamma=1.32$, gas constant $R^{*}=518.3 \mathrm{~J} \bullet \mathrm{kg}^{-1} \bullet \mathrm{K}^{-1}$, viscosity $\mu=0.00001 \mathrm{~Pa} \bullet \mathrm{s}$, the inlet gas pressure and temperature $\left(P_{\text {inlet }}, T_{\text {inlet }}\right)=(115 \mathrm{MPa}, 300 \mathrm{~K})$, and the radius of the basic structural unit $R=0.1 \mathrm{~m}$.

\begin{tabular}{|c|c|c|c|c|}
\hline \multirow[t]{2}{*}{ Fig. no. } & \multirow{2}{*}{$\begin{array}{c}\text { Inner radius of basic structural unit } \\
\qquad \mathrm{r}_{0}(\mathrm{~m})\end{array}$} & \multirow{2}{*}{$\begin{array}{c}\text { Flow length } \\
\text { L(m) }\end{array}$} & \multicolumn{2}{|c|}{ roughness } \\
\hline & & & $\mathrm{r}_{\min }$ & $\mathrm{b}$ \\
\hline \multirow{3}{*}{3,4} & 0.001 & 1 & 0 & 0 \\
\hline & 0.002 & 1 & 0 & 0 \\
\hline & 0.005 & 1 & 0 & 0 \\
\hline \multirow{3}{*}{5,6} & 0.001 & 1 & 0 & 0 \\
\hline & 0.002 & 5 & 0 & 0 \\
\hline & 0.005 & 10 & 0 & 0 \\
\hline \multirow{2}{*}{8,9} & 0.001 & 1 & 0 & 0 \\
\hline & 0.001 & 1 & 0.00098 & 2 \\
\hline \multirow{3}{*}{10,11} & 0.001 & 1 & 0.000998 & 2 \\
\hline & 0.001 & 1 & 0.000998 & 4 \\
\hline & 0.001 & 1 & 0.000998 & 20 \\
\hline \multirow{3}{*}{12,13} & 0.001 & 1 & 0.00099 & 1 \\
\hline & 0.001 & 1 & 0.00098 & 1 \\
\hline & 0.001 & 1 & 0.0009 & 1 \\
\hline \multirow{3}{*}{14,15} & 0.001 & 1 & 0.00098 & 2 \\
\hline & 0.001 & 2 & 0.00098 & 2 \\
\hline & 0.001 & 4 & 0.00098 & 2 \\
\hline
\end{tabular}




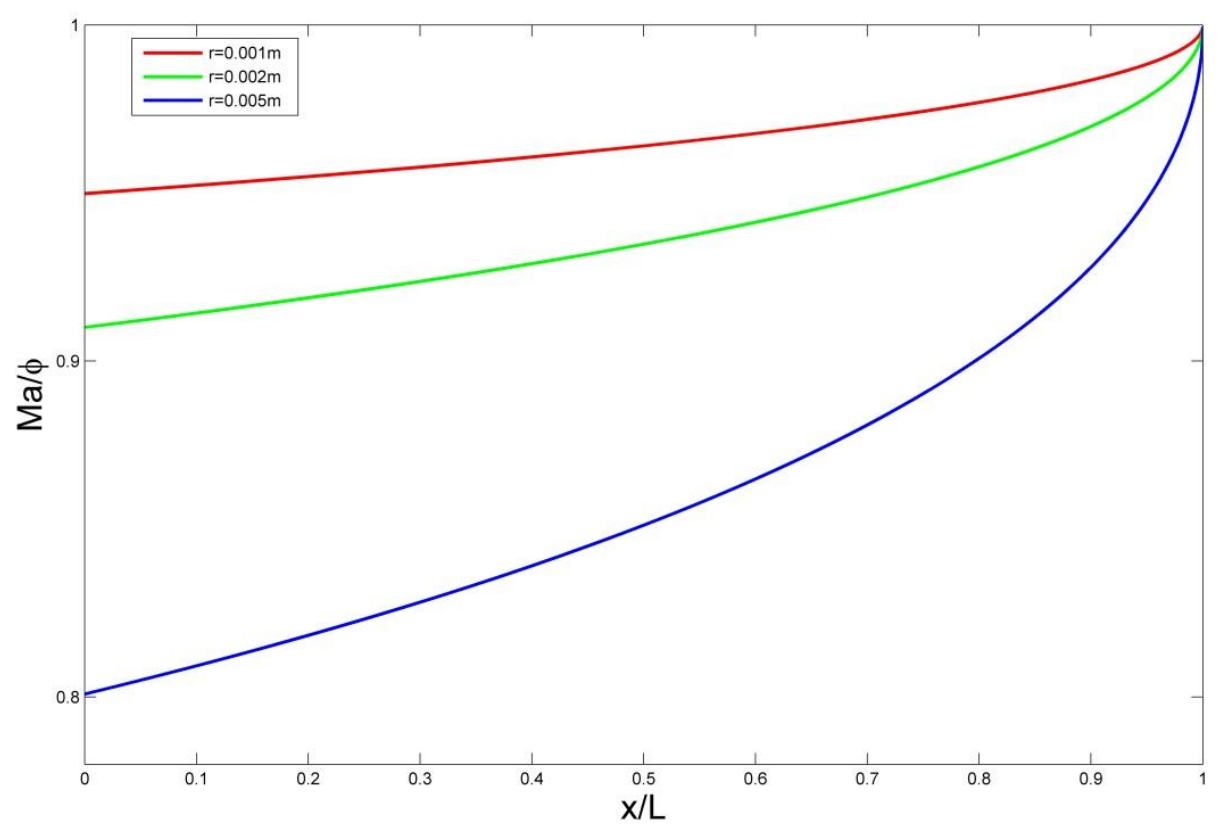

Fig. 3 The Mach number-to-porosity ratio distribution along the dimensionless flow distance at the choking condition.

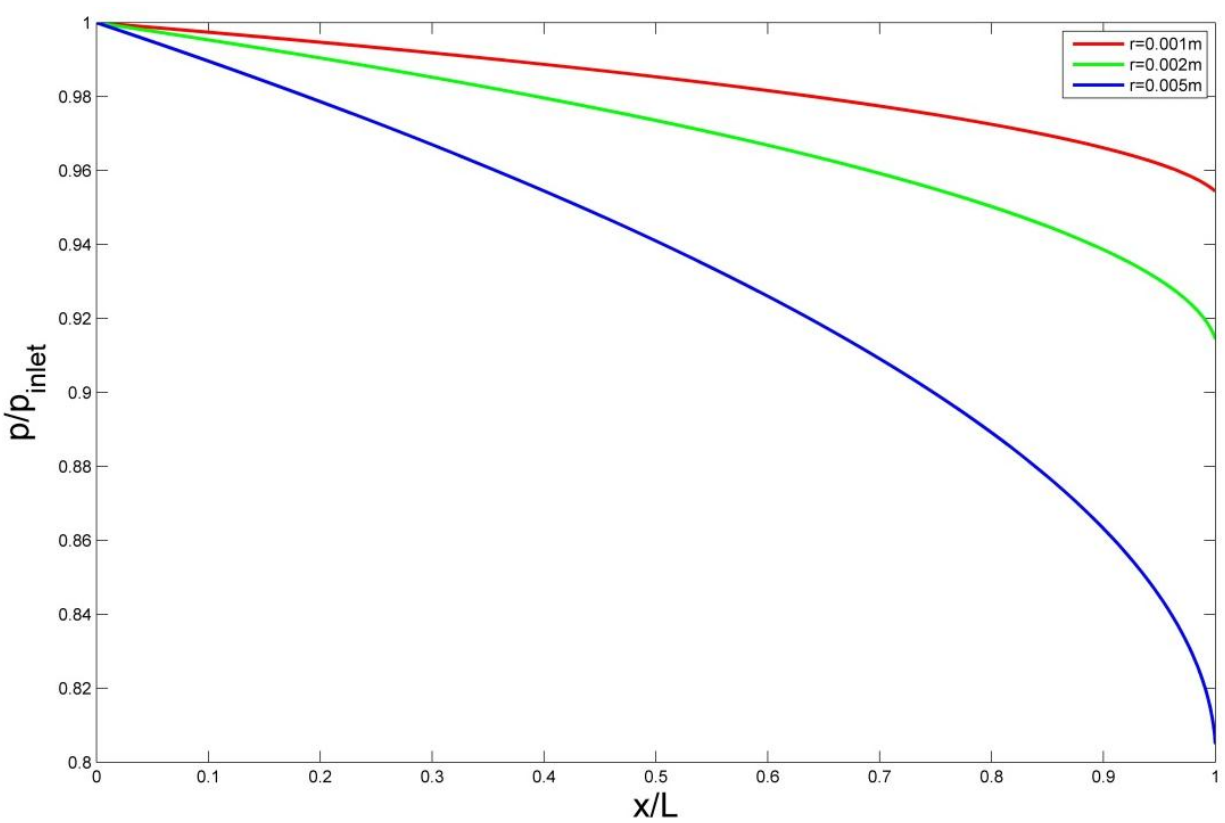

Fig. 4 The dimensionless pressure distribution along the dimensionless flow distance at the choking condition. 


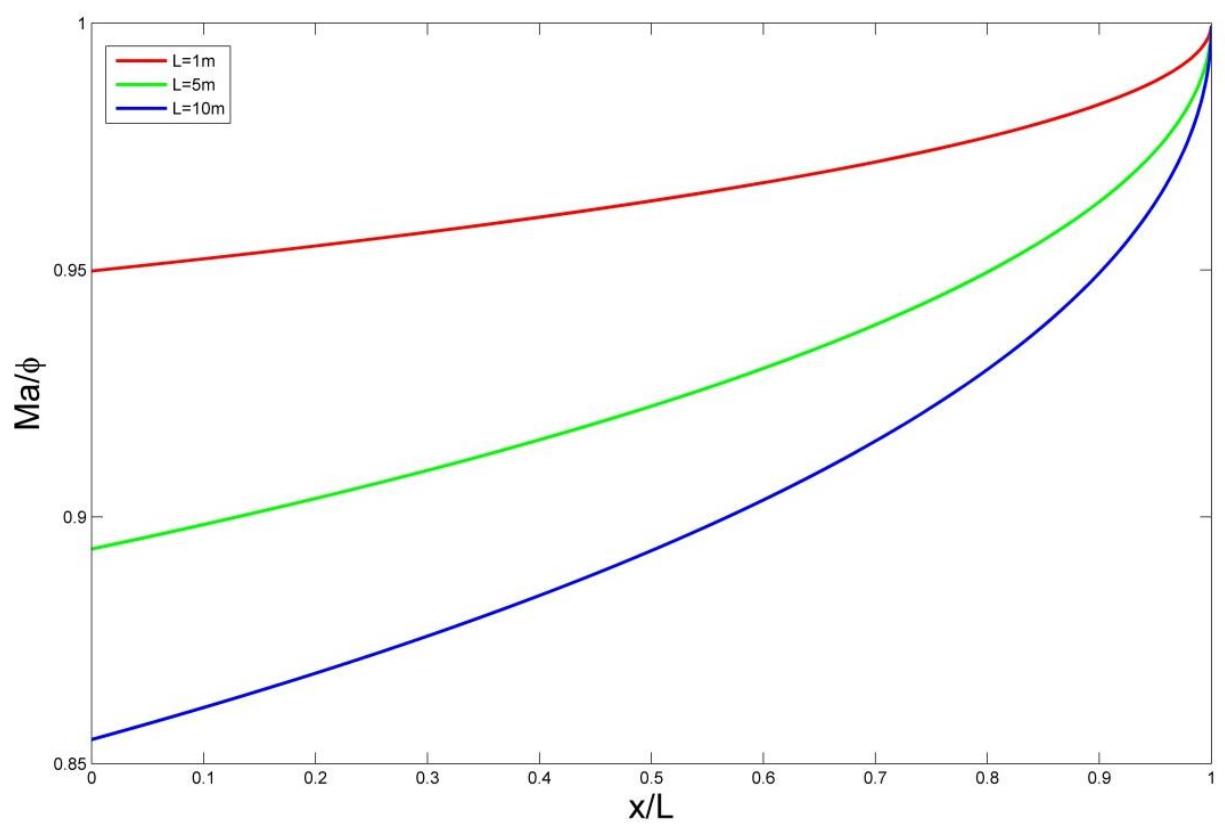

Fig. 5 The Mach number-to-porosity ratio distribution along the dimensionless flow distance at the choking condition

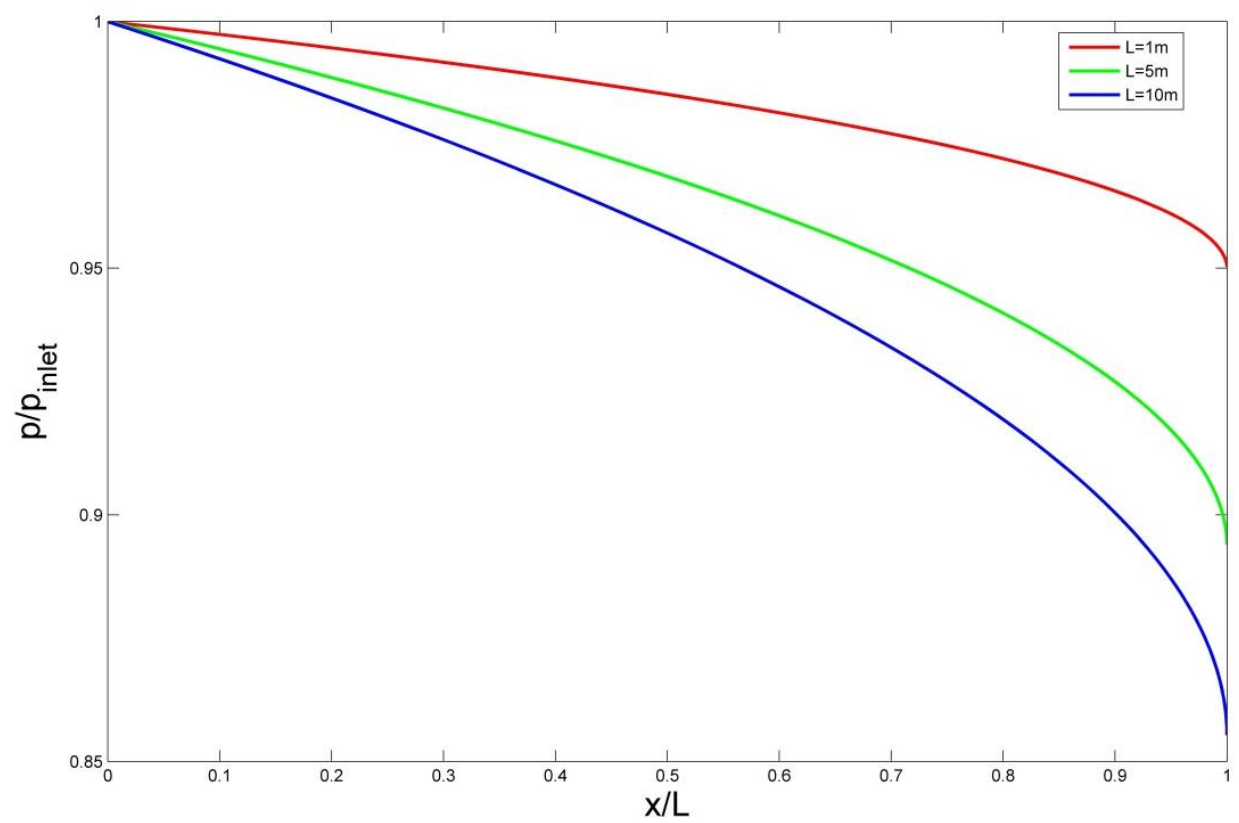

Fig. 6 The dimensionless pressure distribution along the dimensionless flow distance at the choking condition

\subsection{Choked flow in the basic structural unit with roughness in the $\mathrm{x}$ direction}

We are concerned with an axisymmetric basic structural unit having a cosinusoidal 
pore throat profile as depicted in Fig. 7. In an analogous manner, Beresnev (2009) defined a capillary channel profile. The cosinusoidal profile is defined by the following equation.

$$
r(x)=r_{\min }\left[1+\frac{1}{2}\left(\frac{r_{0}}{r_{\min }}-1\right)(1+\cos 2 \pi b x)\right]
$$

where $r_{\min }$ and $r_{0}$ are the minimum and the maximum radius, and $b$ is the reciprocal of spatial period. $r_{\min }$ and $b$ reflect the roughness.

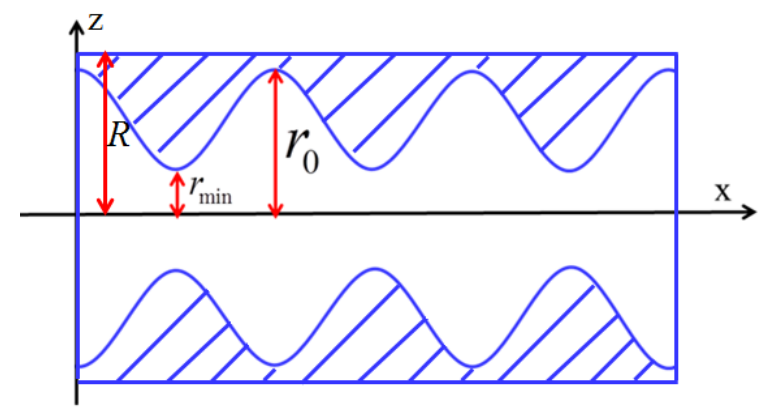

Fig. 7 Cross section of basic structural unit parallel to the flow direction

Combining equation (1), equation (8), equation (9), and equation (13), we can obtain the governing equation of a basic structural unit with roughness.

$$
\begin{aligned}
\frac{d M a^{2}}{d x} & =\frac{\gamma\left(2 \phi^{2} M a^{4}+(\gamma-1) M a^{6}\right)}{\left(\phi^{2}-M a^{2}\right)}\left(\frac{\mu A}{\kappa G}+c_{F} \kappa^{-0.5}\right) \\
& +\frac{2 \phi^{2} M a^{2}+(\gamma-1) M a^{4}}{\left(\phi^{2}-M a^{2}\right) r(x)} 2\left(r_{0}-r_{\text {min }}\right) b \pi \sin (2 \pi b x)
\end{aligned}
$$

Thus, when the inlet pressure is given, we can still search for an appropriate inlet Mach number, at which the outlet Mach number equals the porosity and then we can obtain the Mach number distribution along the basic structural unit. The accompanying pressure distribution is determined from the following equation.

$$
p(x)=\frac{p_{\mathrm{inl} \mathrm{et}} M a_{\mathrm{inl} \mathrm{\textrm { } \mathrm { t }}} r_{0}^{2}}{M a(x) r^{2}(x)} \sqrt{R^{*}} \sqrt{\frac{T_{0}}{1+0.5^{*}(\gamma-1) M a^{2}(x)}}
$$

where $T_{0}$ is the stagnation temperature of the gas.

Firstly, the size of the flow distance and the radius of the basic structural unit are 
fixed with $\mathrm{L}=1 \mathrm{~m}$ and $\mathrm{R}=0.1 \mathrm{~m}$, while the first model is $\mathrm{r}=0.001 \mathrm{~m}$ and the second one is $r(x)=0.001 \times(0.99+0.01 \cos (2 \pi x) \mathrm{m}$. When the two models have the same entrance pressure and temperature in the basic structural unit, the Mach number distribution and accompanying pressure distribution towards the flow direction can be obtained at the choking condition. Fig. 8 shows that the choking condition in the first model occurs at the exit, but the choking condition in the second model occurs at the narrowest inner radius in the basic structural unit. In Fig. 9, it is obvious that a lower pressure drop is required for a rough basic structural unit to become choked.

Secondly, the size of the flow distance and the minimal inner radius of the basic structural unit are fixed, and the values of $b$ (defined as the reciprocal of the spatial period) in the three models are 2, 4 and 20, respectively. In the same way, Figs. 10 and 11 show the Mach number distribution and the accompanying pressure distribution along the flow direction for each of these three basic structural units when the flow is choked. Fig. 10 shows that the choking conditions in the three models all occur at the narrowest inner radius near the exit. In Fig. 11, the exit pressures in these models are 112.96 MPa, 113.03 MPa and 113.21 MPa at the choking condition. It can be seen that a lower pressure drop is required for the flow to become choked in the basic structural unit with a shorter spatial period. Thus, the choked flow occurs first in the basic structural unit with more complicated roughness.

Thirdly, the reciprocal of spatial period and the flow distance are fixed, and the minimal inner radii of the basic structural units in the three models are $0.00099 \mathrm{~m}$, $0.00098 \mathrm{~m}$ and $0.0009 \mathrm{~m}$, respectively. Fig. 12 shows that all choking conditions in the three models occur at the narrowest inner radius near the exit. From Fig. 13, it can be seen that a lower pressure drop is required for the flow to become choked in the basic structural unit with a smaller inner radius. Thus, the flow first becomes choked in the basic structural unit with more complicated roughness.

Lastly, Figs. 14 and 15 show the Mach number distribution and accompanying pressure distribution towards the flow direction for each of these three basic structural units when the flow is choked. In each of these basic structural units, the reciprocal of 
spatial period and the minimal inner radius of basic structural unit are held constant and the values of $L$ in the three models are $1 \mathrm{~m}, 2 \mathrm{~m}$ and $4 \mathrm{~m}$, respectively. From Figs. 14 and 15, it can be seen that a lower pressure drop is required for the flow to become choked in the same rough structural unit with a shorter flow distance.

From the discussion above, it can be seen that the choking condition first occurs at the position of smallest porosity near the exit. For the basic structural unit with roughness, the choked flow first occurs in the basic structural unit with more complicated roughness and shorter flow distance.

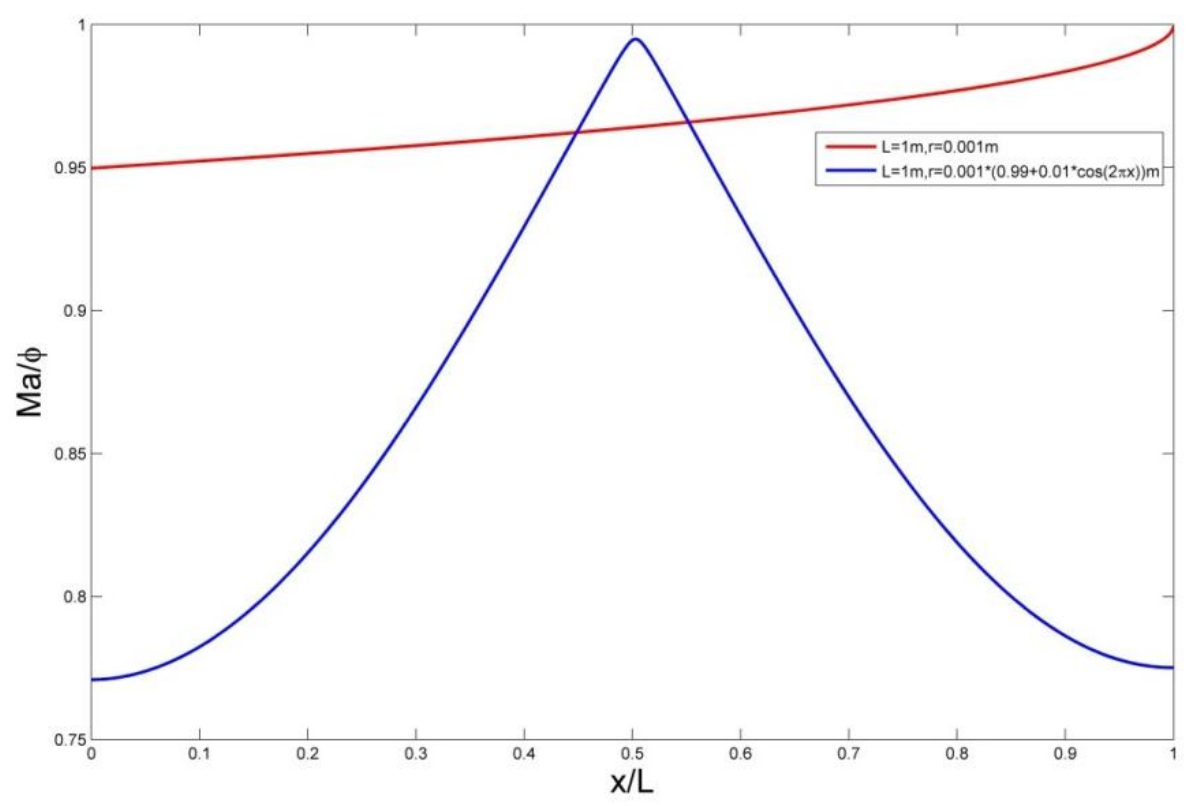

Fig. 8 The Mach number-to-porosity ratio distribution along the dimensionless flow distance at the choking condition 


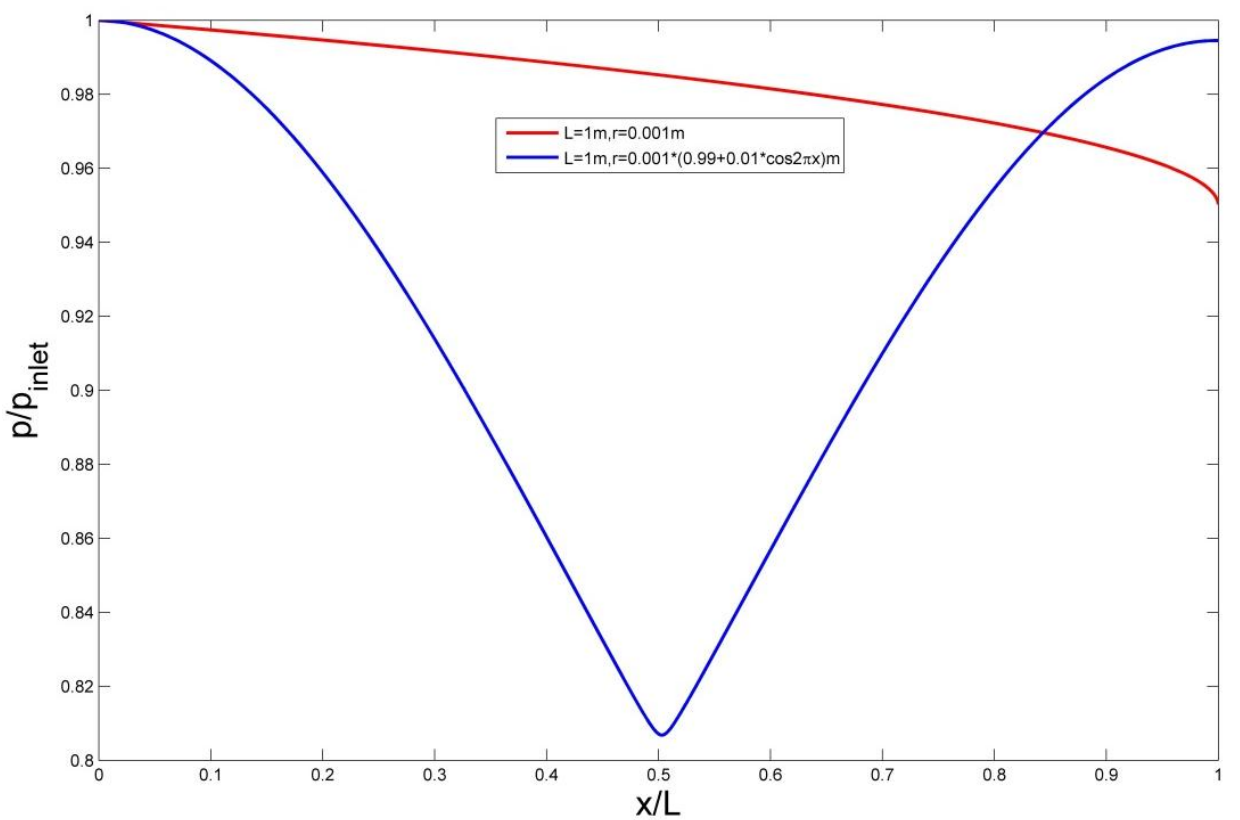

Fig. 9 The dimensionless pressure distribution along the dimensionless flow distance at the choking condition

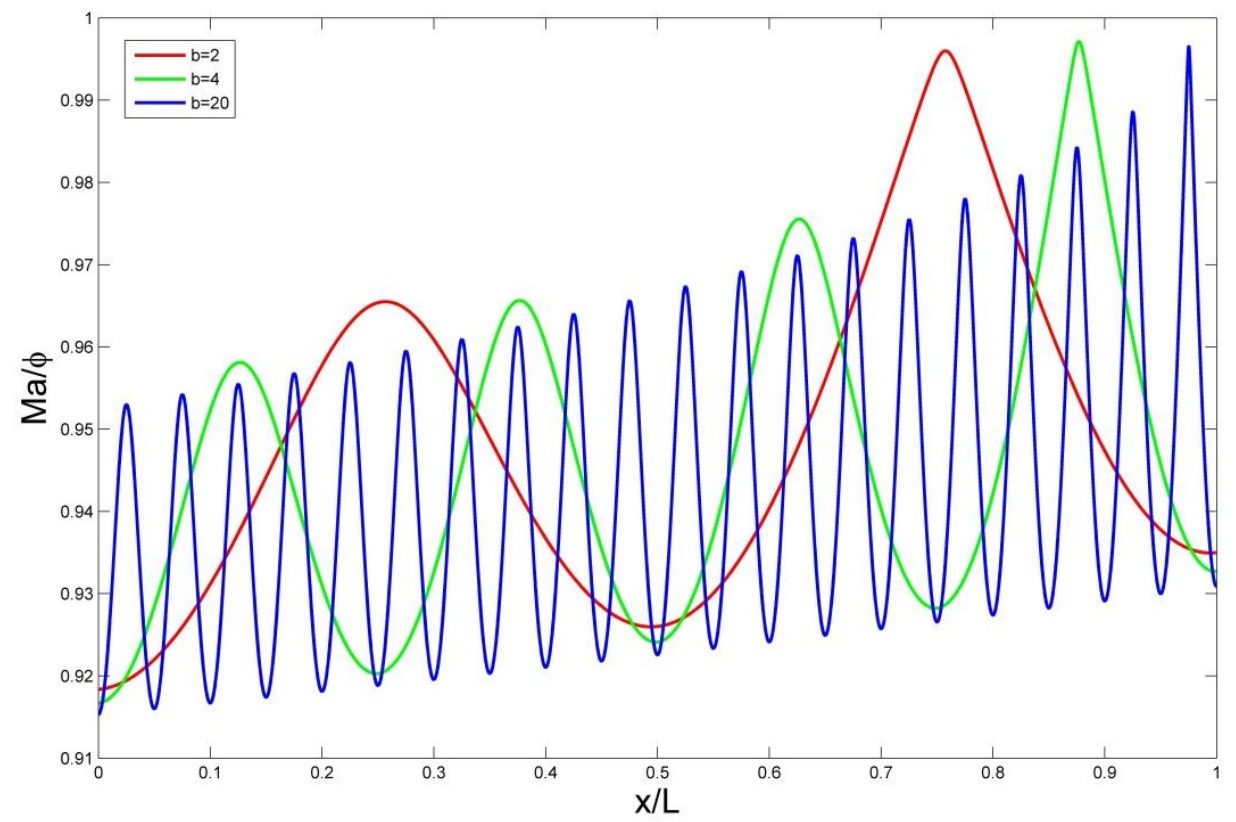

Fig. 10 The Mach number-to-porosity ratio distribution along the dimensionless flow distance at the choking condition 


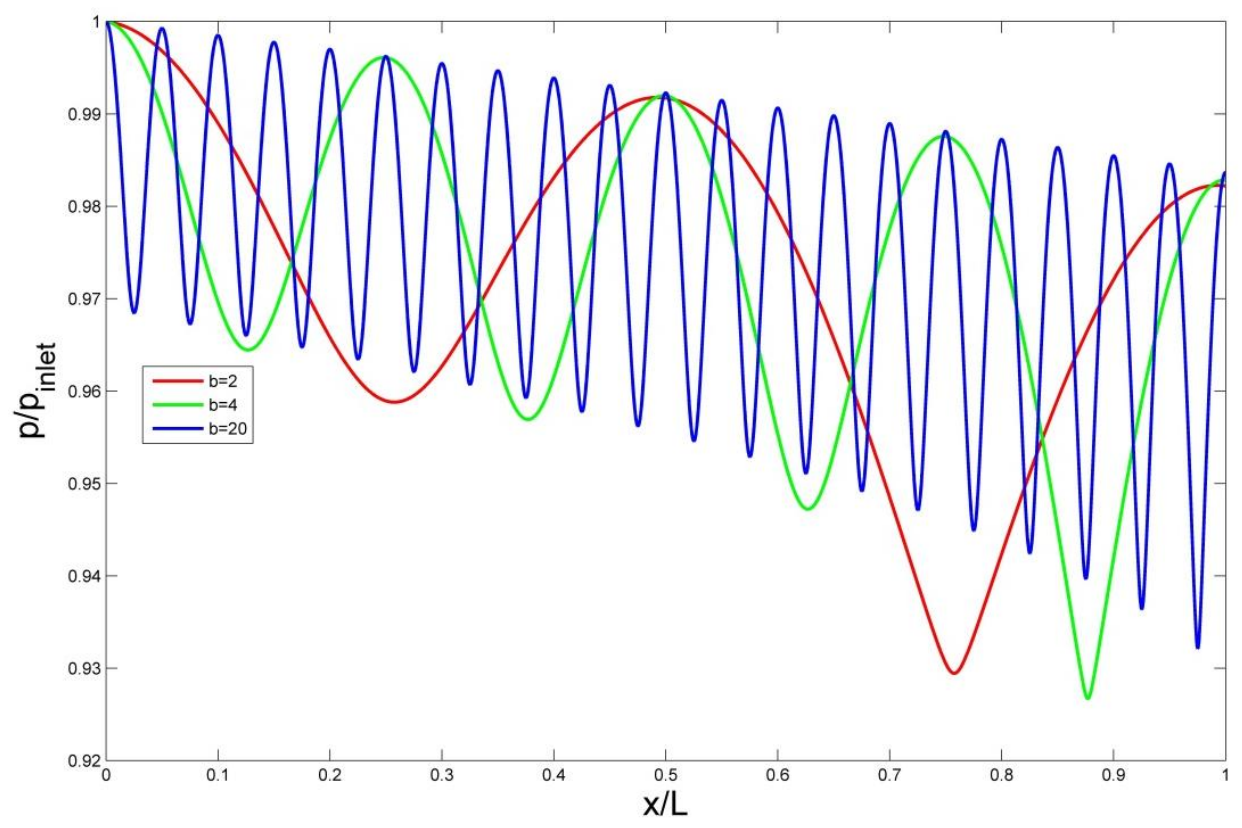

Fig. 11 The dimensionless pressure distribution along the dimensionless flow distance at the choking condition

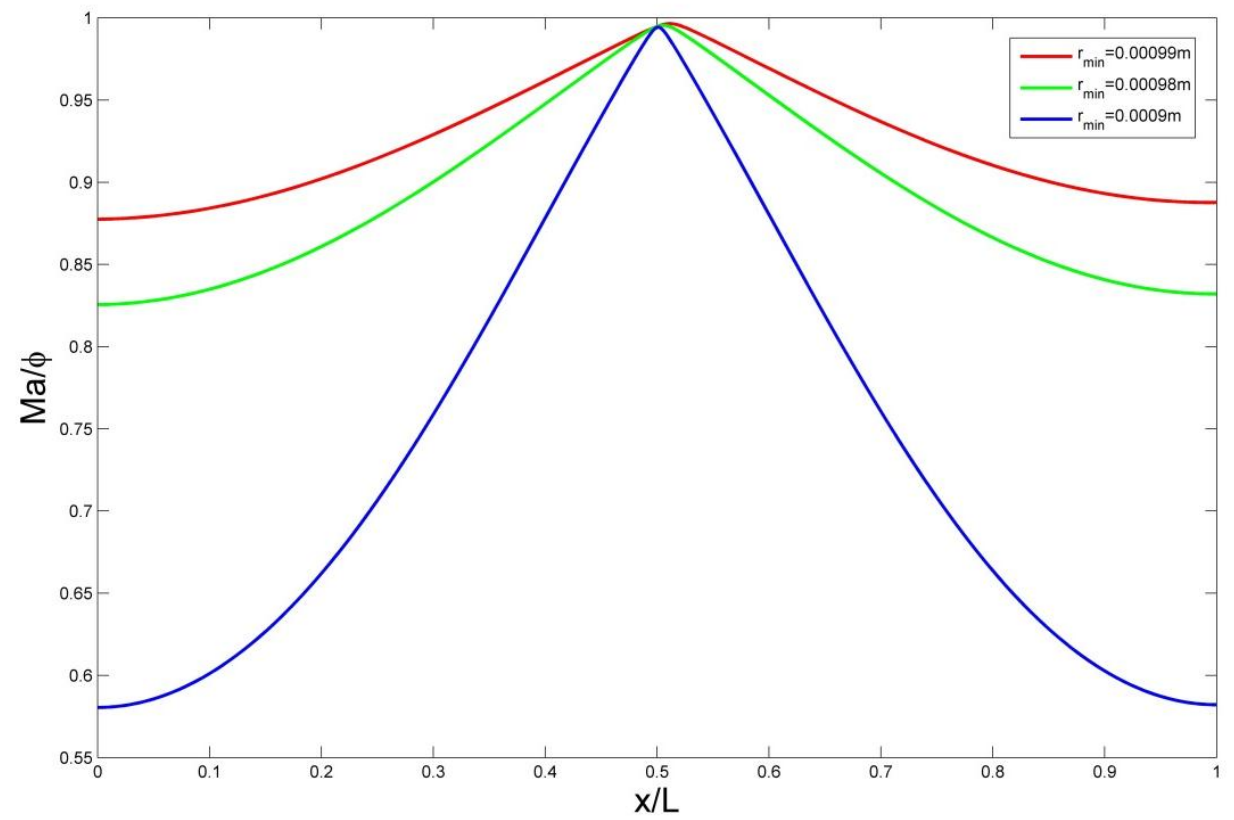

Fig. 12 The Mach number-to-porosity ratio distribution along the dimensionless flow distance at the choking condition 


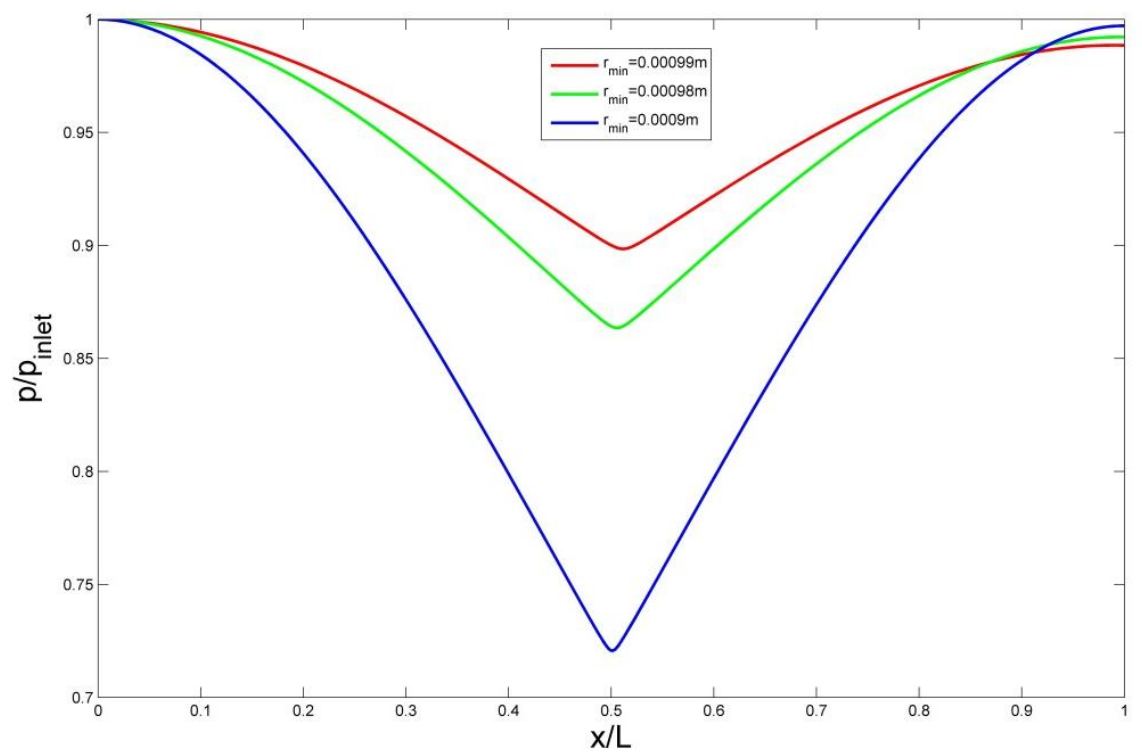

Fig. 13 The dimensionless pressure distribution along the dimensionless flow distance at the choking condition

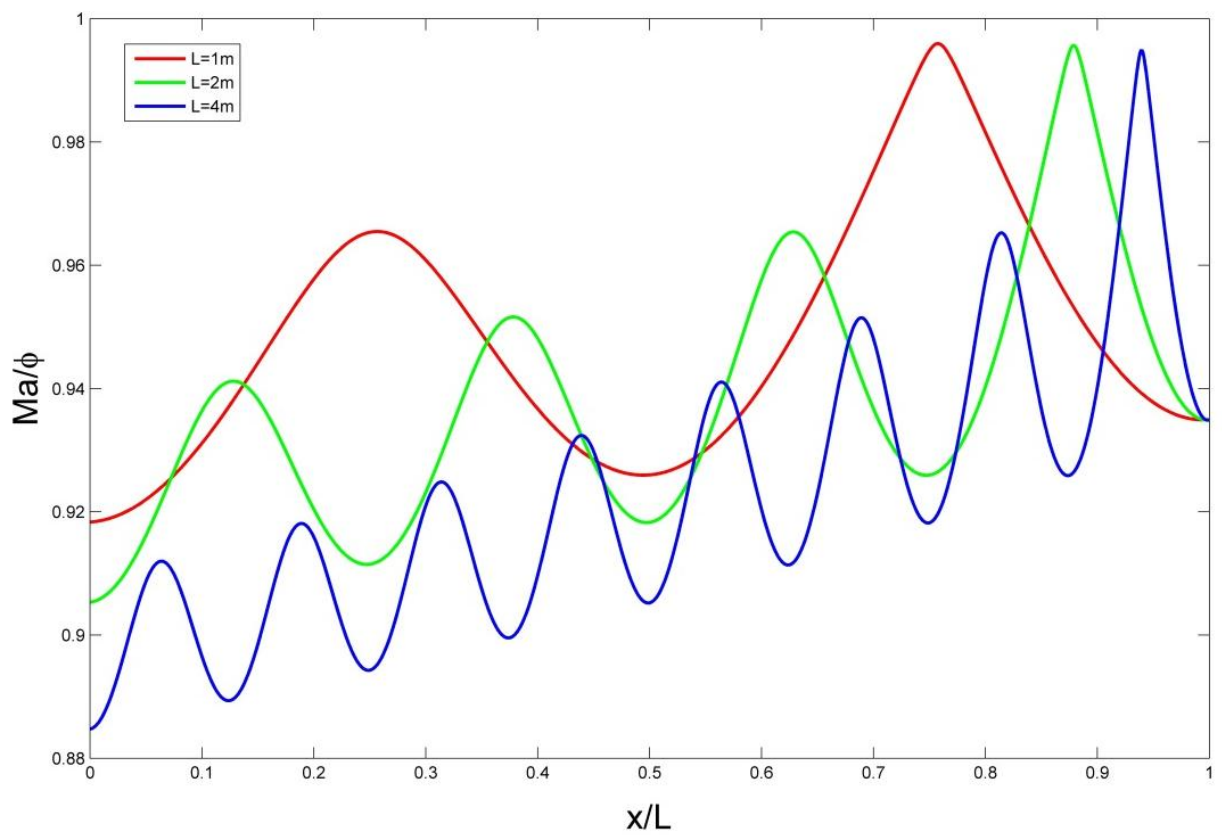

Fig. 14 The Mach number-to-porosity ratio distribution along the dimensionless flow distance at the choking condition 


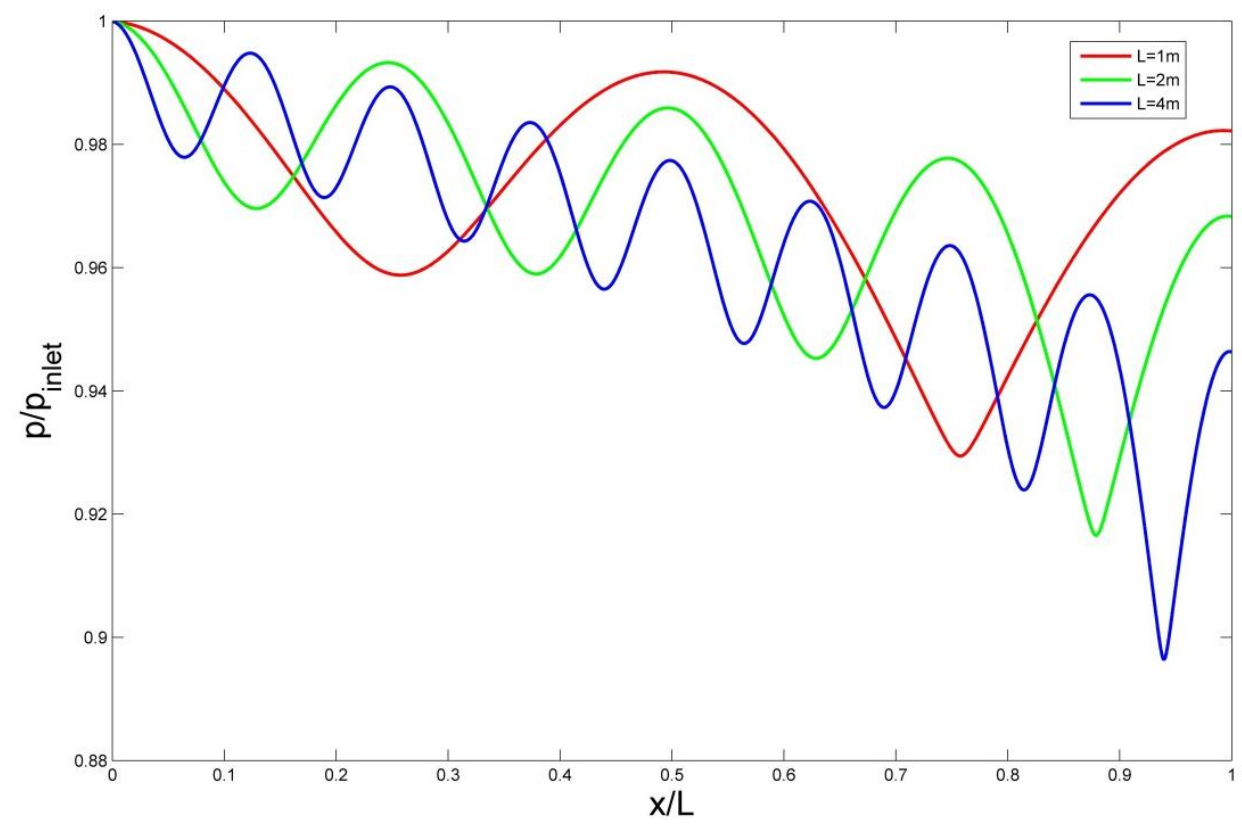

Fig. 15 The dimensionless pressure distribution along the dimensionless flow distance at the choking condition

\section{Analogy of flow in a basic structural unit and in a pipe without wall thickness}

As mentioned in the introduction, many researchers investigated the choked flow in a pipe without wall thickness at the microscopic scale (Meyer and Smith, 1985; Kodres, 1994; Yuan, et al., 2016). Using overbars for the variable quantities of this pipe flow, the moment equation with acceleration is as follow:

$$
\frac{d \bar{p}}{d x}+\bar{\rho} \frac{-d \bar{v}}{d x}+\frac{\bar{\rho}(\bar{v})^{2}}{\bar{r}} f=0
$$

where $\bar{p}$ is the pressure of the gas, $\bar{\rho}, \bar{v}$ are the density and velocity of the gas, respectively, $\bar{r}$ is the radius of the pore throat, and $f$ is a friction factor.

Combining equation (1), equation (8) and equation (16), the governing equation of an adiabatic pipe flow is

$$
\frac{d M^{2}}{d x}=\frac{\left(2 M^{2}+(\gamma-1) M^{4}\right)}{\left(1-M^{2}\right)}\left(\gamma M^{2} \frac{f}{-}-\frac{1}{\bar{A}} \frac{d \bar{A}}{d x}\right)
$$

where the Mach number is $M=\frac{\bar{v}}{\sqrt{\gamma R^{*} T}}$. 
A friction factor of the following form is obtained (Schlichting and Gersten, 2003)

$$
f=f_{1}+f_{t}
$$

where $f_{1}=16 / \operatorname{Re}, f_{t}=c_{1}+c_{2}\left(q / 2 r^{*}\right)^{n}$, Re is the Reynolds number for flow in a pipe and is expressed by $\operatorname{Re}=\frac{2 \rho^{*} v^{*} r^{*}}{\mu^{*}}, c_{1}, c_{2}$ and $n$ are universal constants and $q$ is the effective wall roughness (Meyer and Smith, 1985).

Substituting for $f_{1}$ and $f_{t}$, the friction factor can be written as

$$
f=\frac{8 \mu^{*}}{\rho^{*} v^{*} r^{*}}+c_{1}+c_{2}\left(q / 2 r^{*}\right)^{n}
$$

The intrinsic permeability and porosity of a pipe which is like a cylindrical capillary are, respectively (Chukwudozie, 2012),

$$
\begin{aligned}
& \kappa=\frac{1}{8} r^{2} \\
& \phi=1
\end{aligned}
$$

Substituting equation (20) and equation (21) into equation (14), the governing equation of a basic structural unit flow can be written as

$$
\frac{d M a^{2}}{d x}=\frac{\left(2 M a^{2}+(\gamma-1) M a^{4}\right)}{\left(1-M a^{2}\right)}\left(\gamma M a^{2} \frac{F}{r}-\frac{1}{A} \frac{d A}{d x}\right)
$$

where $F=\frac{\mu r}{\kappa \rho v}+r c_{F} \kappa^{-0.5}$. The coefficient $c_{F}$ can be expressed as (Beavers, 1973)

$$
c_{F}=0.55\left(1-5.5 \frac{d}{2 r}\right)
$$

where $d$ is the diameter of the spheres which implies the roughness of the pore throats.

The empirical equation (23) can be written as

$$
c_{F}=c_{3}-c_{4} \frac{d}{2 r}
$$

Thus, the parameter $F$ in equation (22) is: 


$$
F=\frac{8 \mu}{\rho v r}+c_{3}-c_{4} \frac{d}{r}
$$

Both of these terms are analogous, that is, the term $f$ (equation (19)) in the governing equation of a basic structural unit flow and the term $F$ (equation (25)) in the governing equation of a basic structural unit. The distinction of both terms is in their universal constants, which are mainly caused by turbulent flow. Therefore, the governing equation (17) in a pipe without wall thickness is similar to the governing equation (22) in a pipe with finite wall thickness which is discussed in the previous sections. It can be easily verified that if the basic structural unit is considered as a pipe without wall thickness, i.e., a single pore throat, the obtained result is the same as the result of the previous sections, due to the similar governing equation.

The friction factor has a significant effect on the outlet pressure in macroscopic choked flows (Jiang et al., 2015a, b). In microscopic choked flows, we discuss below the impact of varying the friction factor along the basic structural unit. The parameters used in the following discussions are as follow: adiabatic index $\gamma=1.32$, gas constant $R^{*}=518.3 \mathrm{~J} \bullet \mathrm{kg}^{-1} \bullet \mathrm{K}^{-1}$, viscosity $\mu=0.00001 \mathrm{~Pa} \bullet \mathrm{s}$, the inlet gas pressure and temperature $\left(P_{\text {inlet }}, T_{\text {inlet }}\right)=(115 \mathrm{MPa}, 300 \mathrm{~K}), R=r_{0}=0.01 \mathrm{~m}, L=1 \mathrm{~m}, b=0.5$, and $r_{\min }=0.003 \mathrm{~m} \sim 0.01 \mathrm{~m}$.

The variation of the friction factor $F$ with the dimensionless flow distance $x / L$ and $r_{\min }$ is displayed in Fig. 16. It is clear from Fig. 16 that the friction factor $F$ increases with the dimensionless flow distance $x / L$. As $r_{\min }$ is decreased, the friction factor $F$ increases. As the dimensionless flow distance $x / L$ is increased, the difference of the friction factor $F$ among these curves also increases.

For a varying friction factor, the outlet-to-inlet pressure ratio obtained from the pipe with finite wall thickness is denoted by $P_{F}$. The outlet-to-inlet pressure ratio obtained from the pipe without wall thickness is denoted by $P_{f}$, when the constant 
friction factor is set to $F(x=0)$, i.e., $f=F(x=0)$. Fig. 17 shows the difference in the outlet pressure between the constant friction factor flow and the varying friction factor flow at the microscopic choking condition. The horizontal axis shows $\frac{r_{\min }}{r_{0}}$, and the vertical axis shows $\frac{P_{f}-P_{F}}{P_{f}}$. Obviously, the outlet pressure $P_{F}$ for the varying friction factor is lower than the outlet pressure $P_{f}$ for the constant friction factor. The difference in the outlet pressure between the varying friction factor model and the constant friction factor model increases as $r_{\min }$ is decreased, i.e., the variation in the friction factor can have a significant effect on the outlet pressure in microscopic choked flows. Therefore, the variation of the friction factor cannot be ignored in choked flow.

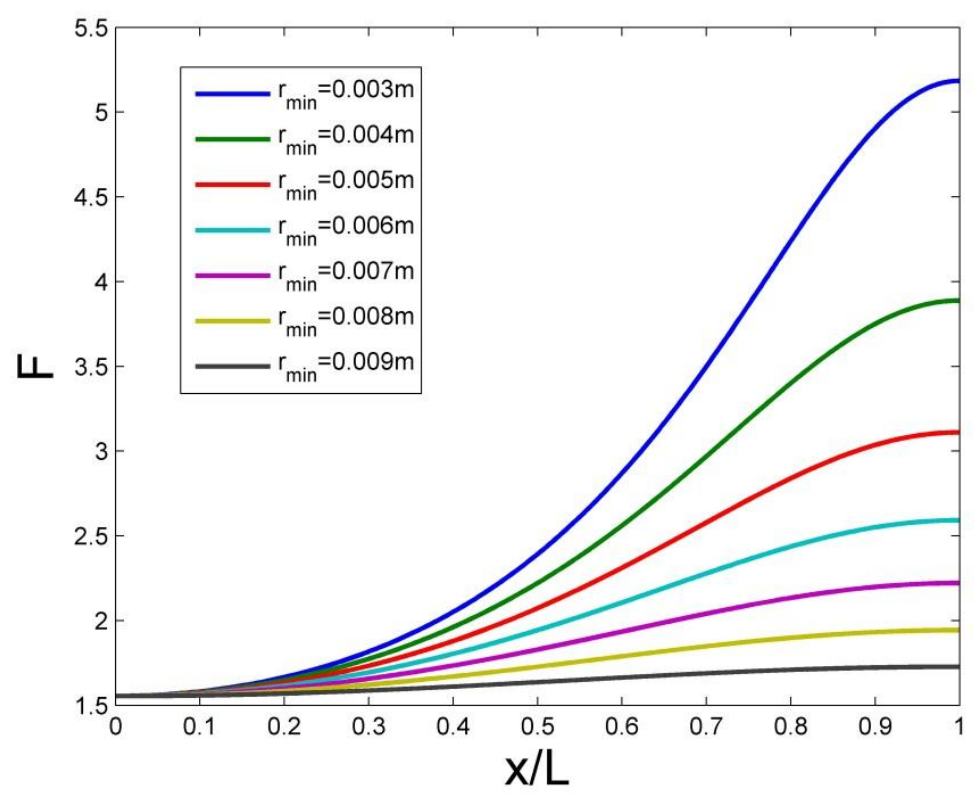

Fig. 16 Friction factor profile with decreasing $r_{\min }$ 


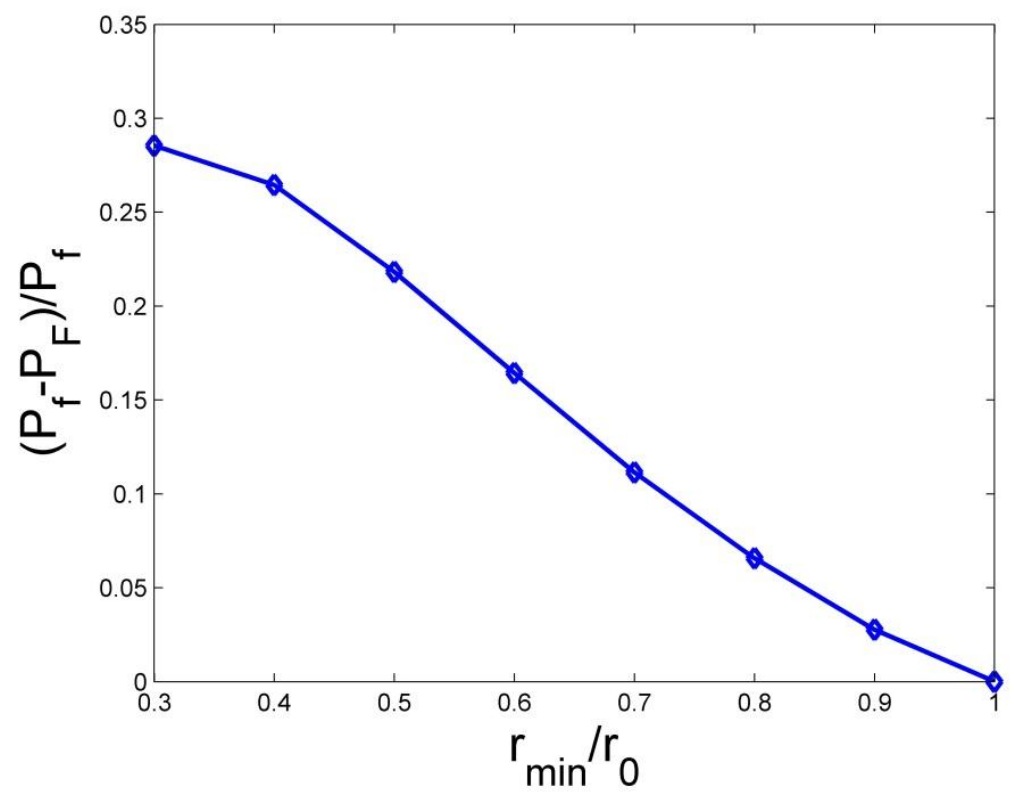

Fig. 17 Difference of the outlet pressure for the constant friction factor and the varying friction factor with different $r_{\text {min }}$

\section{Conclusions}

In this paper, we investigated the choking condition for a high-speed compressible gas flow in a basic structural unit at a microscopic scale. The major findings are as follows:

(1) The substantial choking condition of macroscopic flow in nonhomogeneous porous media only can be obtained by analyzing the basic structural unit. The pressure drop obtained from the basic structural unit flow is lower than that obtained from the corresponding macroscopic nonhomogeneous porous medium flow.

(2) For the basic structural unit without roughness, the choked flow occurs first at the basic structural unit with a lower porosity and shorter flow distance.

(3) The choked flow in the basic structural unit with roughness requires a lower pressure drop than that in the basic structural unit without roughness.

(4) For the basic structural unit with roughness, the choking condition occurs first at the smallest porosity or permeability position near the exit, and the choked flow occurs first in a basic structural unit with more complicated roughness and shorter 
flow distance.

(5) For microscopic choked flows, the outlet-to-inlet pressure ratio under the effect of varying friction is substantially lower than that under the effect of constant friction.

\section{Acknowledgment}

The authors are grateful for the support provided by the Chinese National Science Foundation (No. 51374171, No. 51404198).

\section{References}

Beresnev, I.A., Li, W., Vigil, D.R., 2009. Condition for break-up of non-wetting fluids in sinusoidally constricted capillary channels. Transport in porous media. 80(3):581-604.

Coulaud, O., Morel, P., Caltagirone, J.P., 1988. Numerical modelling of nonlinear effects in laminarflow through a porous medium. Journal of Fluid Mechanics. 190: $393-407$

Ciarletta, M., Straughan, B., 2006. Poroacoustic acceleration waves. Proceedings of the Royal Society of London A: Mathematical, Physical and Engineering Sciences. 462(2075): 3493-3499.

Chukwudozie, C.P., Tyagi, M., Sears, S.O., White, C.D., 2012. Prediction of non-Darcy coefficients for inertial flows through the castlegate sand stone using image-based modeling. Transport in porous media. 95(3): 563-580.

Du Plessis, J.P., 1994. Analytical quantification of coefficents in the Ergun equation for fluid friction in a packed bed. Transport in porous media. 16(2), 189-207.

De Ville, A., 1996. On the properties of compressible gas flow in a porous media. Transport in porous media. 22(3), 287-306.

Du Plessis, J.P., Masliyah, J.B., 1988. Mathematical modeling of flow through consolidated isotropic porous media. Transport in porous media. 3(2), 145-161.

Emanuel, G., Jones, J.P., 1968. Compressible flow through a porous plate. 
International Journal of Heat and Mass Transfer. 11(5): 827-836.

Hayes, R. E., Afacan, A., Boulanger, B., 1995. An equation of motion for an incompressible Newtonian fluid in a packed bed. Transport in porous media. 18(2), 185-198.

Handren, P., Pearson, C.M., Kullman, J., 2001. The impact of non-Darcy flow on production from hydraulically fractured gas wells. SPE Production and Operations Symposium. Society of Petroleum Engineers.

Joseph, D.D., Nield, D.A., Papanicolaou G., 1982. Nonlinear equation governing flow in a saturated porous medium. Water Resour. Res. 18(4): 1049-1052.

Jin, Y., Chen, K.P., Chen, M., 2011. Development of tensile stress near a wellbore in radial porous media flows of a high pressure gas. International Journal of Rock Mechanics \& Mining Sciences. 48, 1313-1319.

Jin, Y., Chen, K.P., Chen, M., 2012a. Highly compressible porous media flow near a wellbore: effect of gas acceleration. Journal of Fluids Engineer - Transactions of the ASME. 134(1), 011301.

Jin Y., Chen, K.P., Chen, M., Grapsas, N., 2012b. Gas expansion-induced acceleration effect in high-pressure gas flows near a wellbore. Journal of porous media. 15(4):317-328.

Jiang, H., Chen, M., Jin, Y., Chen, K., 2015a. Importance of Gas Acceleration Near the Wellbore in Radial Compressible Porous Media Flows for a Vertical Gas Well. Transport in Porous Media. 110(1): 127-140.

Jiang, H., Chen, M., Jin, Y., Chen, K., 2015b. Gas expansion-induced acceleration effect of a highly compressible gas flow in porous media. Journal of Porous Media. 18(8): 825-834

Jiang, H., Chen, M., Jin, Y., Chen, K., 2015c. Analytical modeling of acceleration-induced conductivity damage in a propped hydraulic fracture of a high-pressure gas well. Journal of Natural Gas Science and Engineering. 26: 185-192.

Kodres, A.C., 1994. Flow parameter approach to modeling the flow of heated gases through high resistance porous media. Transport in porous media. 15(3), 
229-249.

Levy, A., Sorek, S., Ben-Dor, G., Bear J., 1995. Evolution of the balance equations in saturated thermoelastic porous media following abrupt simultaneous changes in pressure and temperature. Transport in porous media. 21(3), 241-268.

Meyer, B.A., Smith, D.W., 1985. Flow through porous media: comparison of consolidated and unconsolidated materials. Industrial \& engineering chemistry fundamentals. $24,360-368$.

Ma, H., Ruth, D.W., 1993. The microscopic analysis of high Forchheimer number flow in porous media. Transport in porous media. 13(2), 139-160.

Nield, D.A., 1994. Modelling high speed flow of a compressible fluid in a saturated porous medium. Transport in porous media. 14(1), 85-88.

Shreeve, R.P., 1968. Supersonic flow from a porous metal plate. AIAA Journal. 6(4): $752-753$.

Skjetne, E., Auriault, J.L., 1999. High-velocity laminar and turbulent flow in porous media. Transport in porous media. 36(2), 131-147.

Schlichting, H., Gersten, K., 2003. Boundary-layer theory. Springer Science \& Business Media.

Straughan, B., 2008. Stability and wave motion in porous media. Springer, New York.

Van Golf-Racht, T.D., 1982. Fundamentals of Fractured Reservoir Engineering. Elsevier, Amsterdam.

Ward, J.C., 1964. Turbulent flow in porous media. Journal of the Hydraulics Division. 90(5), 1-12

Wang, X., 2012. Key issues in high-performance natural gas wells. Journal of Natural Gas Science and Engineering. 6, 37-42.

Wang, C., Li, Z.P., Lai, F.P., 2014. A novel binomial deliverability equation for fractured gas well considering non-Darcy effects. Journal of Natural Gas Science and Engineering. 20, 27-37.

Wang, C., Li, Z.P., Lai, F.P., 2014. A novel binomial deliverability equation for fractured gas well considering non-Darcy effects. Journal of Natural Gas Science and Engineering. 20, 27-37. 
Yuan J., 2013. Compressible flow through a porous medium: choking at pore scale and its implications. Arizona State University.

Yuan, J., Chen, K.P., 2016. Choked gas flow at pore-scale and its implications to production from high-pressure gas wells. Journal of Fluids Engineering. 138(1):14501. 